\section{Sobre la propuesta preliminar del Plan Nacional de CTI 2030 de Argentina}

Resumen: En este artículo se analiza el documento preliminar del Plan de Ciencia y Tecnología 2030 elaborado por el Ministerio de Ciencia, Tecnología e Innovación de Argentina, así como diversas consideraciones y conceptos expresados al respecto por las máximas autoridades del organismo y por un interlocutor calificado. Sobre esta base se concluye que el objetivo primordial del Plan es impulsar un desarrollo científico-tecnológico orientando la producción de conocimientos a cubrir demandas estratégicas, sociales y productivas con participación y perspectiva federal. El plan se propone además fortalecer interconexiones con otros organismos del Estado y con sectores estratégicos de la producción, el trabajo y el desarrollo, para la conformación de agendas conjuntas.

Palabras clave: Política científica, Plan Argentina 2030, sectores estratégicos, agendas.

On the preliminary proposal of the National STI Plan 2030 of Argentina

Summary: This article analyzes the preliminary document of the Science and Technology Plan 2030 prepared by the Ministry of Science, Technology and Innovation of Argentina, as well as various considerations and concepts expressed in this regard by the highest authorities of the organization and by a qualified interlocutor. On this basis, it is concluded that the primary objective of the Plan is to promote scientific-technological development by guiding the production of knowledge to meet strategic, social and productive demands with federal participation and perspective. The plan also proposes to strengthen the interconnections with other State agencies and with strategic sectors of production, work and development, for the formation of joint agendas.

Keywords: Scientific policy, Argentina 2030 Plan, strategic sectors, agendas.

Sobre a proposta preliminar do Plano Nacional de CTI 2030 da Argentina

Resumo: Este artigo analisa o documento preliminar do Plano de Ciência e Tecnologia 2030 elaborado pelo Ministério de Ciência, Tecnologia e Inovação da Argentina, bem como várias considerações e conceitos expressos a esse respeito pelas máximas autoridades da organização e por um interlocutor qualificado. Com base nisso, conclui-se que o objetivo primordial do Plano é promover o desenvolvimento científico-tecnológico por meio do direcionamento da produção de conhecimento para o atendimento de demandas estratégicas, sociais e produtivas com participação e perspectiva federativas. 0 plano também propõe fortalecer as interconexões com outros organismos do Estado e com setores estratégicos de produção, trabalho e desenvolvimento, para a conformação de agendas conjuntas.

Palavras-chave: Política científica, Plano Argentina 2030, setores estratégicos, agendas.

\section{Ciencia Tecnología y Política \\ Año $4 \mathrm{~N}^{\circ} 7$ Noviembre 2021}

\author{
Nápoli, Mariángela \\ Licenciada y Profesora en Letras \\ ICE-UBA, CONICET \\ marar.napoli@gmail.com \\ Naidorf, Judith \\ Dra. UBA. Cs de la Educación \\ IICE-UBA, CONICET \\ judithnaidorf@gmail.com
}

Año 4 NN$^{\circ} 7$ Noviembre 2021

Fecha de recibido: 18/08/2021

Fecha de aprobado: 30/10/2021

https://doi.org/10.24215/26183188e066

https://revistas.unlp.edu.ar/CTyP

ISSN 2618-3188

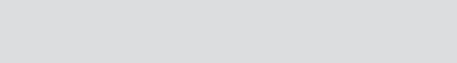

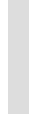


| Ciencia, Tecnología y Política | Año 4 | Nº7 | e066 | Noviembre 2021 | ISSN 2618-3188 | www.revistas.unlp.edu.ar/CTyP |

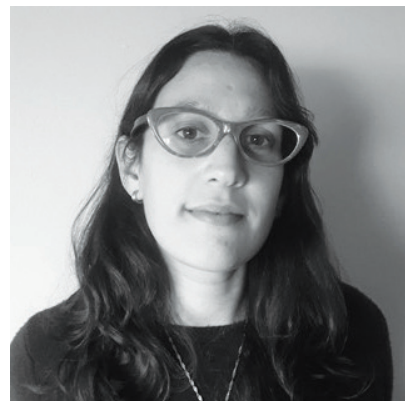

Nápoli, Mariángela

Licenciada y Profesora en

Letras

IICE-UBA, CONICET

marar.napoli@gmail.com

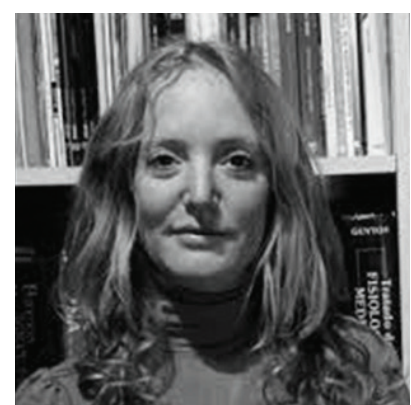

Naidorf, Judith

Dra. en Cs. de la Educación CONICET-ICE-UBA, FFYL-UBA judithnaidorf@gmail.com

\section{Sobre la propuesta preliminar del Plan Nacional de CTI 2030 de Argentina}

Resumen: En este artículo se analiza el documento preliminar del Plan de Ciencia y Tecnología 2030 elaborado por el Ministerio de Ciencia, Tecnología e Innovación de Argentina, así como diversas consideraciones y conceptos expresados al respecto por las máximas autoridades del organismo y por un interlocutor calificado. Sobre esta base se concluye que el objetivo primordial del Plan es impulsar un desarrollo científicotecnológico orientando la producción de conocimientos a cubrir demandas estratégicas, sociales y productivas con participación y perspectiva federal. El plan se propone además fortalecer interconexiones con otros organismos del Estado y con sectores estratégicos de la producción, el trabajo y el desarrollo, para la conformación de agendas conjuntas.

\section{Introducción}

En septiembre de 2020 se lanzó el documento preliminar del Plan de Ciencia y Tecnología 2030 elaborado por el Ministerio de Ciencia, Tecnología e Innovación (MINCYT, 2020). El mismo se define como un ejercicio colectivo de planificación orientado a elaborar el nuevo Plan Nacional de Ciencia, Tecnología e Innovación 2021-2030 (PNCTI) y está inscripto en el proyecto económico, social, productivo y ambiental definido por las políticas gubernamentales.

El anteproyecto se propone alcanzar un desarrollo científico-tecnológico sustentable, con el objetivo de reducir las principales asimetrías del país por causas estructurales e históricamente determinadas: la pobreza, la desigualdad social y la degradación ambiental. En pos de traducir en políticas científicas concretas estos desafíos se procura la "realización de interconexiones con el resto del sector público y otros sectores estratégicos de la producción, trabajo y desarrollo procedentes de ámbitos académicos y de la sociedad civil" (p. 4).

En este artículo se analiza el documento mencionado recuperando algunas nociones de estudios 
| Ciencia, Tecnología y Política | Año 4 | N07 | e066 | Noviembre 2021 | ISSN 2618-3188 | www.revistas.un|p.edu.ar/CTyP |

previos sobre los Planes Bicentenario 2006-2010 (Naidorf, 2011; Marí, Recalde y Fontanals, 2007; Emiliozzi, 2011; Sarthou y Bollini, 2017) y el Plan Argentina Innovadora 2020 (Naidorf, Perrotta, Gómez y Riccono, 2015; Villegas, 2020). En estos trabajos se subrayan, entre otras nociones, por un lado, el desempeño de un papel proactivo del Estado en la creación de una nueva esfera pública basada en el surgimiento y consolidación de redes Estado - actores sociales, que se presenta como necesario para la identificación de problemas críticos. Por otro lado, la relación entre el Plan Bicentenario-Universidades y la posibilidad de coordinar políticas de investigación y de formación de recursos humanos en torno a un conjunto de temáticas previamente acordadas (Loray, 2017; Emiliozzi, 2011). También se señala puntualmente y de carácter más reciente, que el Plan Argentina Innovadora 2020 representó un avance en la identificación y selección de estas áreas estratégicas. Esto se implementó a través de instrumentos concretos de financiamiento a la actividad científica y tecnológica expresados en los Fondos Sectoriales. Además, esta identificación habilitó la discusión respecto de cuáles serían los Recursos Humanos Altamente Calificados que se necesitarían para acompañar dichos sectores. A raíz de ello, se diseñó, entre otros, el instrumento Becas en Temas Estratégicos (BE) dentro del programa de Becas del CONICET, para el cual se realizó una selección de temas y se abrieron convocatorias periódicas, que al día de hoy siguen vigentes (Villegas, 2020).

Cabe aclarar que este primer acercamiento al anteproyecto 2030 se propone describir y analizar algunos conceptos que entendemos como centrales en relación a las propuestas señaladas anteriormente sobre áreas estratégicas y las interconexiones con otros sectores o actores sociales. No se presentará un análisis exhaustivo de las mismas; esto se debe a la obvia concreción a posteriori de esta versión preliminar ya que aún no han desplegado acciones para su implementación ni posee una versión definitiva. Sin embargo, actualmente nos encontramos dialogando con representantes de diferentes provincias para avanzar en un texto que problematice el desarrollo de las premisas aquí señaladas.

En consecuencia, para este trabajo, se abordarán algunas características centrales que se propone asumir el nuevo plan desde la perspectiva de la movilización del conocimiento. Definimos a la movilización del conocimiento (Naidorf y otros, 2014, 2016, 2017 y 2018) como el campo de estudios que problematiza el uso social del conocimiento científico desde tres dimensiones que coinciden con los tres tiempos (antes, durante y después) del proceso investigativo. La primera dimensión de la movilización del conocimiento es la definición de agendas, es decir por qué, para qué y para quién se investiga tal o cual tema. El componente político de esta primera dimensión se corresponde, en el anteproyecto, con el vínculo entre la producción de conocimiento y las demandas sociales y productivas de la sociedad; para ello se explicita la intención de construir en esta primera etapa agendas de CTI con las provincias.

El segundo componente de la movilización del conocimiento son las señales que emite la evaluación del desempeño de investigadores. A grandes rasgos, los criterios utilizados para la evaluación de los proyectos de investigación y del personal que realiza tareas científico-tecnológicas son los heredados de la investigación tradicional que se remonta al paradigma lineal de la producción del conocimiento. Este sistema establecido en torno a 1950 sostiene, entre otras cuestiones, la toma de decisiones establecidas por los pares a la hora de evaluar y legitimar producciones científicas (Invernizzi y Davyt, 2019; Rikap y Naidorf, 2020); el mismo responde a un modelo de 
evaluación por productos, el enfoque más extendido a nivel global, consagrado en los Manuales de Frascati (1963) y de Oslo (1992) de la Organización para la Cooperación y el Desarrollo Económico (OCDE)'. Este tipo de contrato entre científicos y sociedad que se erigió supone un grado de autonomía y autorregulación por parte de los científicos de su propia práctica, que se igualó a garantía de calidad (Alonso, 2018). De esta forma, este modelo enfatiza la calidad académica (anteponiéndolo al criterio de pertinencia social), en la originalidad (por sobre la aplicabilidad) y la consideración de la producción bibliométrica (Martínez Porta, 2014), en detrimento de una valoración a los resultados que no refieren a la producción reflejada solamente en papers. Estas tendencias evaluativas exponen la dificultad de implementar otras formas de evaluar el desempeño orientado a recuperar los aprendizajes que son consecuencia de la interacción con demandantes y con actores extraacadémicos. Posiblemente los cambios institucionales a los que refiere el plan puedan estar orientados hacia el cumplimiento de dicha deuda.

El tercer componente se centra en la efectiva utilización, apropiación y uso del conocimiento científico por el sistema social y productivo. En este plano el anteproyecto hace mención específica a la ciencia orientada a la sociedad. Asimismo, se enfatiza la importancia de la transferencia y la extensión y una referencia general a la circulación del conocimiento. A lo largo del documento se reafirma la necesidad de promover la intervención de la comunidad científica en la resolución de problemáticas sociales y productivas.

Cabe aclarar que el documento discute con el modelo lineal de innovación. Esta visión ingenua -que supone una posición 'ofertista' en la producción de conocimiento- busca ser reemplazada por conceptualizaciones que incorporen elementos e interacciones propios de los procesos de innovación. La noción de 'sistema nacional de innovación', ampliamente discutida (Albornoz, 2009) en el campo ciencia, tecnología y sociedad (CTS), "debe ser el punto de partida para la formulación de las políticas de I+D+i para los países de la región" (Hurtado, 2013: 449). En este trabajo, proponemos focalizar en el primer componente de la movilización del conocimiento, la delimitación de agendas de trabajo, para arribar a algunas definiciones de la actual gestión que postulamos como novedosas, entendiendo que los dos componentes restantes podrán verificarse una vez implementado el proyecto como tal.

\section{Un enfoque federal y concertado de las políticas científicas}

El anteproyecto está pensado como un "ejercicio colectivo de planificación a través de un proceso consultivo, de concertación y planificación a escala nacional" (p. 4). Este proceso se canaliza, en esta primera etapa, a través de los organismos federales y sus representantes en las provincias.

El énfasis en la "concertación" está dado por el arribo a acuerdos para la elaboración de agendas entre las 24 jurisdicciones (23 provincias y CABA) en el marco de la Comisión Asesora para el Plan Nacional de Ciencia, Tecnología e Innovación (CAPLANCYT). En las palabras del Secretario de Planeamiento y Políticas en Ciencia, Tecnología e Innovación²:

denota un gran esfuerzo de diálogo ante el objetivo de transformar y planificar en función de los múlti-

\footnotetext{
1 Ver, entre otros, Cátedra Libre Ciencia, Política y Sociedad (2019).

${ }^{2}$ Entrevista realizada el 8 de abril de 2021 al Secretario de Planeamiento y Políticas en Ciencia, Tecnología e Innovación, en adelante "Entrevista".
} 
| Ciencia, Tecnología y Política | Año 4 | Nº7 | e066 | Noviembre 2021 | ISSN 2618-3188 | www.revistas.unlp.edu.ar/CTyP |

ples y variados intereses que atraviesan al país

Representar esta heterogeneidad implica el reconocimiento del impacto profundo en la organización social existente de las políticas públicas sobre las que se aplica (Hurtado, Bianchi y Lawyer, 2017).

Según el informe realizado por Josefina Vaca y Hugo Berozzi (2020), en cuanto a la distribución de las partidas por provincias y jurisdicciones en ciencia y técnica, se señala que en el año 2019, como se muestra en el gráfico 1, las mismas se distribuyeron de la siguiente manera.

El gráfico 1 refleja las asimetrías que el PNCIT 2030 pretende abordar al contemplar los intereses y las necesidades de cada región. Como sostienen Cao y Vaca (2006), desde fines del siglo XIX el emprendimiento agroexportador de base pampeana ocupó el centro de la dinámica nacional, generando una brecha con el resto de los territorios que nunca pudo cerrarse. En Argentina, los principales organismos destinados a diseñar políticas e instrumentos para la ciencia y la tecnología se crearon en la segunda mitad de la década de 1950 y el $70 \%$ de esas instituciones se concentraron en el área central (Vaca, 2017). El desafío del nuevo gobierno no solo acarrea las problemáticas estructurales, sino problemas acentuados por el gobierno anterior (2015-2019) a partir de una distribución de fondos sin criterios entre los organismos de Ciencia y Técnica en las provincias, sin una orientación política clara y un gran desequilibrio entre fondos (Entrevista). En el anteproyecto se refleja la intención de retomar el concepto de federalismo y apostar a retomar el diálogo perdido entre los representantes de cada provincia.

Para abordar esta problemática, en la nueva gestión se propuso una reunión con representantes del Consejo Federal de Ciencia y tecnología (COFECYT) conformada por especialistas de cada provincia (ministros/as, secretarios/as, dependiendo del grado que le han otorgado) (Entrevista). Desde el primer encuentro se comunicó la decisión de que el modelo sería "de abajo hacia

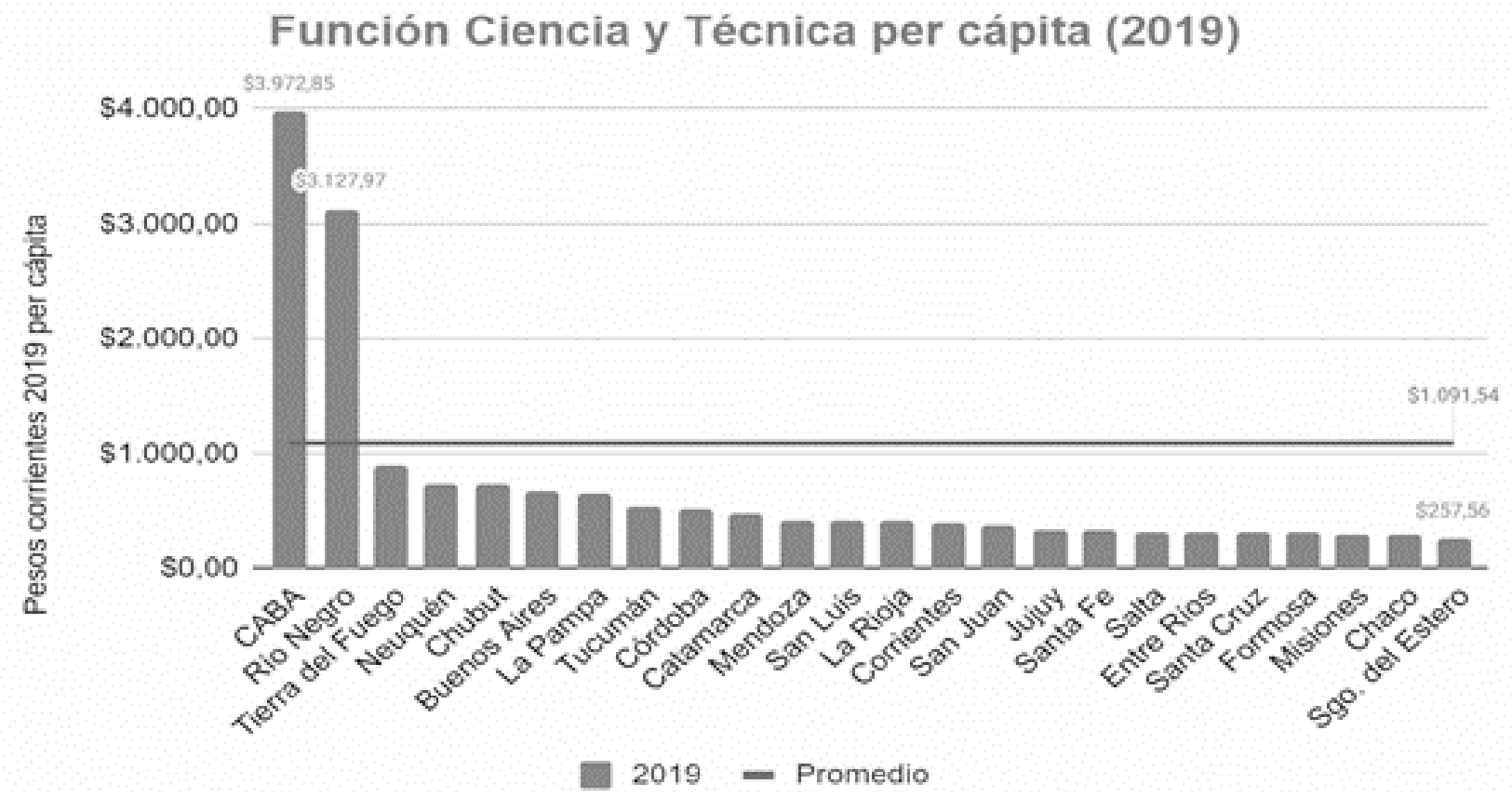

Gráfico 1. Distribución de las partidas presupuestarias en Argentina, para la función ciencia y técnica por provincias y jurisdicciones. Año 2019. Fuente: Informe realizado por Josefina Vaca y Hugo Berozzi como asesores de la actual Diputada Nacional, Claudia Bernazza. 
arriba", como característica central del proceso de construcción colectiva de sus principales contenidos. Producto de esa reunión, se diseñaron diferentes espacios de intercambio de experiencias federales para debatir y definir intereses de cada provincia y región, tal como señaló el Ministro de Ciencia, Tecnología e Innovación en una entrevista: El COFECYT tiene un rol estratégico para garantizar el programa de federalización y revertir las asimetrías. Ahí vamos a discutir cómo se van a aplicar los fondos previstos en la Ley de Financiamiento (Salvarezza, Bilmes \& Liaudat, 2021). Asimismo, desde la página de difusión del COFECYT se señaló que:

El Ciclo de Intercambio de Experiencias Federales es un espacio de diálogo interprovincial organizado por la Subsecretaría de Federalización de la Ciencia, Tecnología e Innovación del MINCYT en conjunto con el COFECYT que busca fortalecer institucionalmente las áreas gubernamentales que gestionan las políticas de Ciencia, Tecnología e Innovación en el territorio, compartiendo experiencias y buenas prácticas de gestión. ${ }^{3}$

Algunos de los enunciados que tuvieron lugar en dichos encuentros lo constituye la experiencia relatada de UBACTEC y diversas Unidades de Vinculación Tecnológica incluida la de INVAP, cuya directora afirmó que:

la ciencia y tecnología con impacto significa, por un lado, tecnología inclusiva y sustentable, es decir, que tenemos que pensar no en una tecnología donde viene el tecnólogo y le dice a la población lo que necesita, sino que se trata de una co-construcción entre ambas partes que debe ser sostenible en el tiempo. Tenemos que trabajar co-creando con la gente que está en territorio, construyendo confianza y con diálogo de saberes.

Para este propósito, se realizaron reuniones virtuales cada 15 días durante todo el año 2021, en las cuales se relevaron experiencias ${ }^{4}$ y se pensaron colectivamente las necesidades y demandas de cada provincia, así como la conformación de bloques de trabajo por región: NOA, NEA, PATAGONIA Y CENTRO5.

Por último, cabe destacar que tanto con el Plan Argentina contra el Hambre ${ }^{6}$, como con las convocatorias a diversos proyectos orientados ${ }^{7}$ se proponen, de forma general, que "el modelo debe adaptarse a un mundo donde la esperanza de vida se extiende, y la demanda en aumento de alimentos y energía ejerce una presión creciente sobre los recursos naturales" (p. 20). Estas dos cuestiones principales oscilan entre una concepción local que refuerza una demanda de atención a necesidades puntuales, así como demandas globales surgidas de acuerdos internacionales. Estas últimas se presentan en el plan, en sintonía con los 17 objetivos de desarrollo sostenible de la Organización de Naciones Unidas (ONU) a los que Argentina suscribe y apuntan a la articulación interministerial. Las demandas sociales, productivas y ambientales se materializan en el documento como parte de un proceso que pretende impulsar un proceso de diálogo y consulta para pensar el desarrollo de las actividades de CTI

\footnotetext{
${ }^{3}$ Extraído de: www.argentina.gob.ar/noticias/comenzo-el-ciclo-de-intercambio-de-experiencias-federales

${ }^{4}$ Véase sobre ello: www.argentina.gob.ar/noticias/agencias-provinciales-de-ciencia-y-tecnologia-en-el-segundo-encuentro-del-ciclo-de

${ }^{5}$ Véase: https://www.argentina.gob.ar/noticias/ciclo-de-intercambio-de-experiencias-federales

6 Véase: https://www.argentina.gob.ar/argentina-contra-el-hambre

7 Véase, por caso: https://www.argentina.gob.ar/noticias/el-mincyt-destinara-70-millones-de-pesos-para-financiar-proyectos-de-tecnologia-e y https://www.argentina.gob.ar/ciencia/sact/impactar entre otros.
} 
para los próximos diez años y cómo éstas darán respuesta a las mismas, en el marco de un proceso de cambio estructural a nivel nacional y regional. (p. 28). Este cambio estructural enunciado es modificar la matriz productiva del país, a partir de un Estado protector y emprendedor (p. 41). En este sentido se supone una concepción de la gestión de la política pública guiada por las metas de agregación de valor, generación de divisas, creación de puestos de trabajo de creciente calificación, promoción de la inclusión y la igualdad social (p. 5).

\section{Responder a las demandas nacionales: la conformación de agendas de trabajo}

El paradigma lineal imperante para la producción del conocimiento ha sostenido que la toma de decisiones está en manos de "los pares" a la hora de legitimar las producciones científicas (Rikap y Naidorf, 2020). Este modelo establecido en los países centrales y luego trasladado a América Latina se guía bajo la concepción insumo-producto: el Estado debe garantizar los recursos para el funcionamiento de la ciencia básica, que decantará en las investigaciones aplicadas y los desarrollos tecnológicos que redundarán en beneficios sociales y económicos. Esta oscilación entre un modelo ofertista y políticas errantes frente a temas definidos como estratégicos, ha dificultado, en los últimos años, la consolidación de políticas públicas que efectivamente orientaran la ciencia hacia la sociedad. En palabras de Diego Hurtado, a través del anterior Plan Argentina Innovadora 2020 se trató de reconectar, de revertir el proceso de desindustrialización que no permitía generar demandas ni sentar agendas (Entrevista), aunque este Plan se discontinuó y abandonó con el cambio de gobierno nacional en 2015. De esta forma, el anteproyecto intenta recuperar premisas orien- tadas al desarrollo nacional y al cambio de la matriz productiva del país a partir del reconocimiento de las demandas territoriales.

Entre1990 y los 2000, diversos trabajos (Etzkowitz, 1990; Gibbons et al., 1994, Sarewitz, 2006) han puesto en discusión el modelo lineal y ofertista, señalando el posible surgimiento de un nuevo paradigma científico orientado a un nuevo contrato entre ciencia y sociedad. Desde esta perspectiva los conceptos de "demanda" y su relación con el de "relevancia socio productiva" se constituyen como ejes nodales de las políticas científicas. Los mismos están asociados a modelos que buscan atender a una necesidad o propósito identificable en el entorno social, económico, productivo, político, cultural, ambiental, etc., para traducirlos en objetivos de las políticas científicas orientados a la resolución de problemas.

El rol de las ciencias sociales y humanas en la reflexión acerca de qué tipo de Ciencia y Técnica se necesita y qué modelo de país subyace en su elección de política científica, así como el tipo de políticas públicas concretas que posibilitarían su concreción (Hurtado, 2014) necesita seguir siendo discutido. De manera general el texto se propone abordar este desafío en el apartado "Agendas de las Ciencias Sociales" como aspecto transversal. Así como las categorías equívocas de "demanda, agentes sociales y desarrollo" requieren ser analizadas durante el devenir de la planificación del proyecto definitivo, surgen los siguientes planteamientos:

Los representantes de las provincias: ¿manifiestan los múltiples intereses a partir de amplias consultas? ¿Qué agentes se sumarán a esas mesas de trabajo para decidir qué acciones se requieren? ¿Cuáles de estas problemáticas estructurales pueden traducirse en proyectos que garanticen el trabajo entre los diversos sectores del país 
(sector público y privado así como sectores de la sociedad civil)? Para responder estas cuestiones, hemos comenzado a entrevistar a funcionarios de diferentes provincias sobre los agentes sociales consultados para elaborar las posturas representativas de sus respectivos territorios.

Otro aspecto central a debatir y que recorre de manera implícita y explícita el anteproyecto lo constituye la propia categoría de "adaptación", a partir de los emergentes surgidos en la pandemia. En el texto se manifiesta la necesidad de aprendizajes en torno a la realización de cambios y adaptaciones en el funcionamiento institucional para hacer frente a las emergencias (...) o a la necesidad de procurar una adaptación a la coyuntura actual de la pandemia y pospandemia en el primer tramo de la planificación (p. 36 y 39). Ahora bien, ¿Cómo se define este concepto de "adaptación"? ¿Debe la ciencia "adaptarse" a las necesidades sociales, en este caso, representadas por los responsables (políticos) de las provincias? ¿Adaptarse implica limitar los horizontes impertinentes que proponen algunos estudios científicos? (Naidorf, 2005). Este interrogante se podrá ir resolviendo en la conformación efectiva de mesas de trabajo y el rol que se le dé a la comunidad académica y a diferentes actores sociales durante las siguientes etapas de elaboración del Plan ${ }^{8}$. Experiencias previas, tales como los procesos consultivos frente a la elaboración de la Ley Nacional de Educación sancionada en 2006 o a las rondas de participación de diferentes actores sociales para el reemplazo de la Ley de Educación Superior (LES) de entre 2007 y 2011, sirven de pautas válidas para legitimar planes de esta envergadura en contraposición con otras experiencias inconsultas tales como la sanción de la LES o la presentación del proyecto UNICABA que generaron un gran rechazo de la comunidad educativa.

Agendas nacionales, territoriales, transversales y de cambio institucional

El anteproyecto plantea la conformación de cuatro clases de agendas:

$\checkmark$ Nacionales: tendrán por objeto la solución de grandes problemas complejos asociados al desarrollo sostenible.

$\checkmark$ Territoriales: harán foco en sectores y temas relevantes para el desarrollo de las regiones.

$\checkmark$ Transversales: están dirigidas a identificar y seleccionar tecnologías con capacidad de impactar en un amplio rango de sectores económicos y sociales, subsectores o eslabones de cadenas de valor.

$\checkmark$ De Cambio Institucional: tiene el propósito de ordenar las medidas a tomar para reforzar y dinamizar progresivamente los ecosistemas de aprendizaje, desarrollo e innovación.

Cada una de las agendas se propone abordar un aspecto particular desde las necesidades generales más amplias y urgentes a las puntuales de cada región y de cada provincia o municipio. Las Agendas Nacionales se subdividen en dos tipos:

1. Agendas para la Acción: tienen un horizonte de corto y mediano plazo. Su objetivo es desplegar conocimientos y recursos de asesoramiento científico y tecnológico disponibles. También desarrollar conocimientos para dar soluciones a problemáticas concretas y canalizar demandas.

2. Agendas Estratégicas: estas agendas se materializarán en los desafíos o retos nacionales, y serán organizadas en torno a grandes problemas

\footnotetext{
8 Ver presentación de los puntos centrales del PNCTI 2030 del 8 de noviembre en el CCK: https://www.argentina.gob.ar/noticias/alberto-fernandez-las-sociedades-ricas-son-las-que-desarrollan-el-conocimiento-la-ciencia-y.
} 
| Ciencia, Tecnología y Política | Año 4 | N7 | e066 | Noviembre 2021 | ISSN 2618-3188 | www.revistas.unlp.edu.ar/CTyP |

de interés nacional.

Las agendas territoriales también son de dos tipos:

1. Agendas Provinciales: tienen como antecedente el trabajo realizado en el marco del programa de asistencia técnica para la elaboración de agendas de I+D+i provinciales. Allí, se definió por parte de cada jurisdicción un conjunto de 4 a 6 núcleos productivos estratégicos (NPE) en los cuales la CTI pueda contribuir a su desarrollo.

2. Agendas Regionales: están destinadas a la identificación conjunta de temas prioritarios para el desarrollo regional, por parte del MINCyT y de un conjunto de jurisdicciones determinadas, ya sea reunidas en el marco de los Consejos Regionales de CYT (CRECYT) u organizadas de acuerdo a una delimitación estructurada en torno a un tema específico de interés.

Asimismo las agendas transversales se subdividen en:

1. Agendas de Tecnologías Aplicadas a la Producción: implican desafíos en el desarrollo y aplicación de tecnologías para impulsar procesos de aprendizaje, escalamiento e innovación que involucran esfuerzos de gestión para transferir conocimientos.

2. Agendas de Ciencias Sociales y Humanas: buscan comprender y facilitar los procesos sociales asociados a la transformación productiva, así como favorecer el desarrollo con inclusión social. En este marco, se inscriben los estudios de las dimensiones sociales, culturales y políticas de la ciencia, la tecnología y la innovación y su evolución y desarrollo para acompañar el cambio estructural.

Si bien la caracterización de cada agenda es ge- neral resta conocer cómo se relevarán y posteriormente conformarán las mismas. Al respecto se afirma que se propone un modelo al revés: empezar con las demandas de las provincias y generar las agendas provinciales (territoriales). Luego, el Ministerio establece un diálogo con esas demandas para vincularlas con el modelo de gestión actual. Se unifican ciertas demandas a nivel regional para abordar las economías regionales (Entrevista).

Por ende, a partir de estas declaraciones y en sintonía con lo establecido en el anteproyecto, se puede visualizar una doble demanda: por un lado, las establecidas a modo de directrices por la gestión gubernamental nacional; $y$, por otro, las de las provincias. Este recorrido compone una especie de modelo circular de retroalimentación de las mismas. Se señala que hay libertad en cuanto a cómo se conformarán las mesas en cada provincia, pero deben orientarse hacia el rumbo del plan (Entrevista).

Al respecto el ex Ministro Salvarezza afirmó:

Hemos introducido algo que es realmente disruptivo: que la elaboración del plan sea hecha desde las provincias a partir de las metas regionales de desarrollo. Por ejemplo, ¿a qué va a estar apostando el NOA? ¿Al litio? El triángulo de Jujuy, Salta y Catamarca, ¿lo toma como prioridad? O el tema de las energías alternativas, eólicas en el caso de la Patagonia. La idea es poder definir con ellos una agenda, conocer las propuestas para cada región y para cada provincia. (Salvarezza, Bilmes y Liaudat, 2021).

Cabe destacar que también se ha mencionado, en recientes intervenciones de funcionarios ${ }^{9}$, la posibilidad de partir de áreas de conocimiento para la concertación de los principales nodos de

\footnotetext{
9 Véase: https://www.youtube.com/watch?v=igd3p1YdREk
} 
demanda; sin embargo, en pos de reducir las asimetrías en torno a la federalización que esta división continuaría perpetuando, se decidió por la modalidad expuesta anteriormente.

Por último, si bien la noción de co-producción se despliega brevemente en las agendas transversales y a partir de los planes de intervención concertados, no se aclara cómo se generarán los acuerdos y compromisos relativos a las intervenciones, medios y recursos a través de los cuales llevar a cabo su implementación (p. 50) ni cómo se llevará a cabo la materialización de estas demandas a través de convenios con las contrapartes intervinientes en estas definiciones (p. 50).

\section{Conclusiones}

En el anteproyecto presentado en septiembre de 2020 se destaca el concepto de "orientación a la demanda" como eje para la elaboración de planes de intervención concertados que debieran marcar el rumbo hacia la postulación de un cambio de matriz productiva del país. Asimismo, se resaltan tres dimensiones del proyecto gubernamental: la social, la productiva y la ambiental.

Esta concepción propone un cambio estructural progresivo, que fortalecería la cultura institucional y sin duda también la cultura académica (Naidorf y Perrotta, 2016), entendida esta última como los discursos, representaciones, motivaciones, normas éticas, concepciones, visiones y prácticas institucionales de investigadores. Destacamos el debate que está implícito en esta definición y que no supone consensos y armonía, sino puja de intereses. El futuro Plan será producto de una negociación que subyace en la materialización de un texto que establece ambiciosas directrices de políticas científicas y que supone virar el timón hacia un rumbo determinado.
Si bien las aspiraciones señaladas en el anteproyecto en un contexto pandémico resultan relevantes, están repletas de dificultades. Es menester seguir repensando los conceptos de co-producción y el rol de la sociedad civil o de los actores extra-académicos que han sido históricamente relegados a meros espectadores en el proceso de producción del conocimiento. El MINCyT tiene previsto la presentación próximamente de una versión del proyecto definitivo, en la que se podrá analizar qué objetivos y definiciones quedaron finalmente plasmadas en el documento.

\section{Bibliografía}

Albornoz, M. (2009). Indicadores de innovación: las dificultades de un concepto en evolución. CTS: Revista iberoamericana de ciencia, tecnología y sociedad, 5(13), 9-25.

Alonso, M (2018). Hacia una nueva definición de utilidad del conocimiento científico. Un primer análisis de los Proyectos de Desarrollo Tecnológico y Social (PDTS) como política pública en Ciencia y Tecnología en Argentina. Trilogía. Ciencia, tecnología y Sociedad, 9, 79 - 97.

Cao, H.; Vaca, J. (2006). El fracaso del proceso descentralizador argentino. Una aproximación desde la crítica a sus supuestos conceptuales. Nómadas. Critical Journal of Social and Juridical Sciences, 14(2)

Cátedra Libre Ciencia, Política y Sociedad. (2019). La evaluación en ciencia y tecnología en Argentina. Ciencia, tecnología y política, 2(3), 025. https:// doi.org/10.24215/26183188e025

Gibbons, M.; Limoges, C.; Nowotny, H.; Schwartzman, S.; Scott, P.; Trow, M. (1994). La nueva producción del conocimiento. Pomares.

Emiliozzi, S. (2011). Políticas en ciencia y tecnología, definición de áreas prioritarias y universidad 
en Argentina. Revista Sociedad, 29(30), 1-17.

Etzkowitz, H. (1990). The second academic revolution: The role of the research university in economic development. En S. E. Cozzens, P. Healey, A. Rip, J. Ziman (Eds.), The research system in transition (pp. 109-124). Springer.

Hurtado, D. (2013). Techno-political and sectorial culture in a semi-peripheral context: nuclear development in Argentina (1945-1994). Revista iberoamericana de Ciencia, Tecnología y Sociedad (online), 7(21), 163-192.

Hurtado, D. (2014). Surgimiento, alienación y retorno. El pensamiento latinoamericano en ciencia, tecnología y desarrollo. Voces en el Fénix, $n^{\circ} 20$.

Hurtado, D.; Bianchi, M. D.; Lawler, D. (2017). Tecnología, políticas de Estado y modelo de país: el caso ARSAT, los satélites geoestacionarios versus "los cielos abiertos". Epistemología E Historia De La Ciencia, 2(1), 48-71. https://revistas.unc.edu.ar/ index.php/afjor/article/view/18640

Invernizzi, N.; Davyt, A. (2019) Críticas recientes a la evaluación de la investigación: ¿Vino nuevo en odres viejos? Redes, 25(49), 233-252.

Loray, R. (2017). Políticas públicas en ciencia, tecnología e innovación: tendencias regionales y espacios de convergencia. Revista de Estudios Sociales, 62, 68-80. https://dx.doi.org/10.7440/ res62.2017.07

Marí, M.; Recalde, A.; Fontanals, J. (2007). Prospectiva y planificación estratégica en ciencia y tecnología en Argentina. Cuadernos del CENDES, 24(66), 115-125.

Martínez Porta, L. (2014). Universidad y territorio: la universidad como agente de desarroIlo local. [Tesis de Maestría, Universidad de San Andrés]. https://repositorio.udesa.edu.ar/ jspui/bitstream/10908/10905/1/\%5bP\%5d\%-
5bW\%5d\%20T.\%20M.\%20AyPP.\%20Mart\%c3\%adnez\%20Porta,\%20Laura.pdf

Ministerio de Ciencia, Tecnología e Innovación [MinCyT] (Septiembre de 2020). Plan Nacional de Ciencia, Tecnología e Innovación. Documento preliminar. https://www.argentina.gob.ar/sites/ default/files/plan_cti_2030_-_documento_preliminar_septiembre_2020.pdf

Naidorf, J. (2005). La privatización del conocimiento público en universidades públicas. En: P. Gentili (comp.), Espacio público y privatización del conocimiento. Estudios sobre políticas universitarias en América Latina. CLACSO.

Naidorf, J. (2011). Criterios de relevancia y pertinencia de la investigación universitaria y su traducción en forma de prioridades. Revista de Sociología de la Educación-RASE, 4(1), 48-58.

Naidorf, J. (2014). Knowledge utility: from social relevance to knowledge mobilization. Education Policy Analysis Archives, 22(89), 1-31.

Naidorf, J.; Perrotta, D.; Riccono, G.; Gómez, S. (2015). Políticas universitarias y políticas científicas en Argentina pos 2000. Crisis, innovación y relevancia social. Revista Cubana de Educación Superior, 34(1), 10-28.

Naidorf, J.; Vasen, F.; Alonso, M. (2016). Los Proyectos de Desarrollo Tecnológico y Social como política científica. Brazilian Journal of Latin American Studies, 27, 1-21.

Naidorf, J.; Perrotta, D. (2016). Los cambios en la cultura académica frente al cambio de ciclo. Revista del IICE, 39.

Naidorf, J; Alonso, M. (2018). La movilización del conocimiento en tres tiempos. Revista Lusófona de Educação, 39, 81-95.

Rikap, C; Naidorf, J. (2020). Ciencia privatizada en América Latina. Con-Ciencia Social, 2, 57-76. 
Salvarezza, R.; Bilmes, G.; Liaudat, S. (2021). "No volveremos a ser los mismos, asistimos a un cambio de mentalidad en la relación entre ciencia, tecnología y sociedad". Ciencia, Tecnología y Política, 4(6), 051. https://doi.org/10.24215/26183188e051

Sarewitz, D. (2006). Public science and social responsibilities. Development, 49(4), 68-72.

Sarthou, N. F.; Bollini, M. A. (2017). La política y los instrumentos de la cooperación internacional en ciencia y tecnología de la Argentina reciente. Chakiñan, 3, 83-101.

Vaca, J. (2017). Asimetría territorial y desarrollo científico-tecnológico. IADE-Realidad Económica. https://www.iade.org.ar/noticias/asimetria-territorial-y-desarrollo-cientifico-tecnologico

Vaca, J.; Berozzi, H. (Octubre de 2020). Función Ciencia y Técnica de acuerdo a la distribución geográfica [Informe elaborado por el equipo de asesorxs en CTI de la Diputada Nacional Claudia Bernazza, Congreso de la Nación].

Villegas, M. (2020). Procesos de planificación en CTI: el Plan Argentina Innovadora 2020 y sus principales instrumentos: FONARSEC y Becas para Temas Estratégicos [Tesis de Maestría, Facultad Latinoamericana de Ciencias Sociales]. https://repositorio.flacsoandes.edu.ec/xmlui/handle/10469/16309 